\title{
ROS-mediated inactivation of the PI3K/AKT pathway is involved in the antigastric cancer effects of thioredoxin reductase-1 inhibitor chaetocin
}

\author{
Chuangyu Wen ${ }^{1,2}$, Huihui Wang ${ }^{1,3}$, Xiaobin Wu ${ }^{1,2}$, Lu He$^{1}$, Qian Zhou ${ }^{1,2}$, Fang Wang ${ }^{1}$, Siyu Chen ${ }^{4}$, Lanlan Huang ${ }^{1}$, \\ Junxiong Chen ${ }^{1,3}$, Huashe Wang ${ }^{1,2}$, Weibiao Ye ${ }^{5}$, Wende Li ${ }^{4}$, Xiangling Yang $\mathbb{1}^{1}$, Huanliang Liu ${ }^{1,3}$ and \\ Junsheng Peng ${ }^{1,2,6}$
}

\begin{abstract}
Novel drugs are urgently needed for gastric cancer (GC) treatment. The thioredoxin-thioredoxin reductase (TRX-TRXR) system has been found to play a critical role in GC tumorigenesis and progression. Thus, agents that target the TRXTRXR system may be highly efficacious as GC treatments. In this study, we showed that chaetocin, a natural product isolated from the Chaetomium species of fungi, inhibited proliferation, induced $G_{2} / M$ phase arrest and caspasedependent apoptosis in both in vitro and in vivo models (cell xenografts and patient-derived xenografts) of GC. Chaetocin inactivated TRXR-1, resulting in the accumulation of reactive oxygen species (ROS) in GC cells; overexpression of TRX-1 as well as cotreatment of GC cells with the ROS scavenger N-acetyl-L-cysteine attenuated chaetocin-induced apoptosis; chaetocin-induced apoptosis was significantly increased when GC cells were cotreated with auranofin. Moreover, chaetocin was shown to inactivate the PI3KJAKT pathway by inducing ROS generation; AKT1 overexpression also attenuated chaetocin-induced apoptosis. Taken together, these results reveal that chaetocin induces the excessive accumulation of ROS via inhibition of TRXR-1. This is followed by PI3K/AKT pathway inactivation, which ultimately inhibits proliferation and induces caspase-dependent apoptosis in GC cells. Chaetocin therefore may be a potential agent for GC treatment.
\end{abstract}

\section{Introduction}

Gastric cancer (GC) is the fifth most common cancer and the third leading cause of cancer-related death worldwide $^{1}$. The traditional treatment options for GC include surgery and chemotherapy. However, the 5-year

\footnotetext{
Correspondence: Huanliang Liu (liuhuanl@mail.sysu.edu.cn) or Junsheng Peng (pengjsh@mail.sysu.edu.cn)

'Guangdong Provincial Key Laboratory of Colorectal and Pelvic Floor Diseases, Guangdong Institute of Gastroenterology, The Sixth Affiliated Hospital, Sun Yat-sen University, Guangzhou, Guangdong, China

2Department of Gastrointestinal Surgery, The Sixth Affiliated Hospital, Sun Yatsen University, Guangzhou, Guangdong, China

Full list of author information is available at the end of the article. These authors contributed equally: Chuangyu Wen, Huihui Wang Edited by V. Stambolic
}

survival rates of patients receiving these therapies are still very low. The targeting of specific molecules could be an efficient strategy for the treatment of GC. Recently, some novel molecular targeted agents have been used to treat GC, including trastuzumab (which targets the epidermal growth factor receptor) and bevacizumab (which targets the vascular endothelial growth factor). However, the efficiency of these drugs is limited ${ }^{2,3}$. Thus, the identification of new molecular targets and the development of novel targeted therapeutic agents, which can be highly efficacious in treating $\mathrm{GC}$, are urgently needed.

The thioredoxin-thioredoxin reductase (TRX-TRXR) system, which is composed of TRXRs, TRXs and NADPH, plays an essential role in maintaining cellular redox

\section{(c) The Author(s) 2019}

(c) (i) Open Access This article is licensed under a Creative Commons Attribution 4.0 International License, which permits use, sharing, adaptation, distribution and reproduction in any medium or format, as long as you give appropriate credit to the original author(s) and the source, provide a link to the Creative Commons license, and indicate if changes were made. The images or other third party material in this article are included in the article's Creative Commons license, unless indicated otherwise in a credit line to the material. If material is not included in the article's Creative Commons license and your intended use is not permitted by statutory regulation or exceeds the permitted use, you will need to obtain permission directly from the copyright holder. To view a copy of this license, visit http://creativecommons.org/licenses/by/4.0/. 
homeostasis. In eukaryotes, the flavoenzyme TRXRs have two confirmed forms, TRXR-1 and TRXR-2, which are located in the cytoplasm and mitochondria, respectively. TRXRs are the only known TRX-reducing enzymes; they generate reduced TRXs by providing reducing equivalents in an NADPH-dependent reaction. Reduced TRXs function as antioxidants by donating reducing equivalents to reactive oxygen species (ROS) scavenging enzymes such as peroxiredoxins. By regulating cell redox events, the TRX-TRXR system influences various cellular functions including proliferation, differentiation and death ${ }^{4-7}$. However, the TRX-TRXR system has recently been found to be upregulated in a variety of human cancers including gastric, colorectal, lung and liver cancers, and overexpression of specific components of this system is linked to tumor cell proliferation, invasion, metastasis, and drug resistance $^{8-11}$. Inhibition of the TRX-TRXR system abolishes tumor progression ${ }^{12-14}$. As the TRX-TRXR system plays an important role in GC tumorigenesis and progression $^{11,13}$, targeting the TRX-TRXR system may represent an effective GC treatment option.

Chaetocin is a small-molecule thiodioxopiperazine natural product isolated from the Chaetomium species of fungi ${ }^{15,16}$. Recently, some studies have shown that chaetocin has a potent inhibitory effect on cancer cells ${ }^{17-21}$, indicating that chaetocin may be a potential agent for cancer therapy. Molecular mechanisms associated with the anticancer effect of chaetocin are still vague. The inhibition of histone methyltransferase suppressor of variegation 3-9 homolog 1 (SUV39H1), which trimethylates lysine 9 of histone h3, and hypoxia-inducible factor$1 \alpha(\mathrm{HIF}-1 \alpha)$ may be included in the anticancer activity of chaetocin $^{22-24}$. Most importantly, chaetocin was shown to inhibit the activity of TRXR-1 in the cell-free system, which may be related to its anticancer effect ${ }^{25}$. However, the pharmacological effect and underlying mechanism of action of chaetocin in GC cells remains unclear. In the present study, we investigated the antiGC effects of chaetocin both in vitro and in vivo and determined whether chaetocin exerts its anticancer effects in GC by inhibiting TRXR-1.

\section{Materials and methods \\ Cell culture}

Human gastric cancer cell lines HGC-27, AGS, BGC823, SGC-7901 and human embryo kidney cell line HEK293T were purchased from the Culture Collection of the Chinese Academy of Science (Shanghai, China). Human gastric cancer cell lines SNU-216, MKN-45 and human gastric mucosa epithelial cell line GES-1 were obtained as a gift from Professor Ruihua Xu, State Key Laboratory of Oncology in South China, Sun Yat-sen University Cancer Center. HEK-293T cells were maintained in DMEM (Life Technologies, Carlsbad, CA, USA), and all other cell lines were maintained in RPMI 1640 (Life Technologies). All culture media were supplemented with $10 \%$ fetal bovine serum (Life Technologies), 100 units/ml penicillin and $10 \mathrm{mg} / \mathrm{ml}$ streptomycin (Life Technologies). All cells were cultured in a humidified $5 \% \mathrm{CO}_{2}$ atmosphere at $37^{\circ} \mathrm{C}$.

\section{Reagents}

Chaetocin was purchased from Sigma-Aldrich (St. Louis, MO, USA). Chaetocin was resuspended in DMSO at a concentration of $10 \mathrm{mM}$ and was stored at $-20^{\circ} \mathrm{C}$. z-VAD-fmk (Selleck Chemicals, Houston, TX, USA) was resuspended in DMSO at a concentration of $100 \mathrm{mM}$ and was stored at $-20^{\circ} \mathrm{C}$. LY294002 (Selleck Chemicals) was resuspended in DMSO at a concentration of $50 \mathrm{mM}$ and was stored at $-20^{\circ} \mathrm{C}$. N-acetyl-L-cysteine (NAC) (SigmaAldrich) was resuspended in DMSO at a concentration of $0.5 \mathrm{M}$ and was stored at $-20^{\circ} \mathrm{C}$. phospho-histone $\mathrm{h} 3$ (Ser473), phospho-CDK1 (Thr161), PARP, caspase-3, cleaved-caspase-3, caspase-9, cleaved-caspase-9, caspase8, BCL-2, BCL-XL, MCL-1, survivin, XIAP, TRX-1, phospho-AKT (Ser473), AKT and ki-67 antibodies were purchased from Cell Signaling Technology (Beverly, MA, USA). $\beta$-actin and flag tag antibodies were purchased from Proteintech Group (Chicago, IL, USA). Anti-mouse immunoglobulin $\mathrm{G}$ and anti-rabbit immunoglobulin $\mathrm{G}$ horseradish peroxidase-conjugated secondary antibodies were purchased from Sigma-Aldrich.

\section{TRX-1 and AKT-1 overexpression}

A pLV-EF1 $\alpha$-EGFP(2A)Puro vector with TRX-1 insert was purchased from Cyagen Biosciences (Suzhou, Jiangsu, China) and used to stably overexpress TRX-1. Expression, packaging (psPAX2) and envelope (pMD2.G) plasmids were transfected into HEK-293T cells using lipofectamine 3000 (Life Technologies). Lentiviral particles were collected from the supernatant and used to infect HGC-27 and AGS cells. Stable cell lines were established by puromycin selection. A pENTER-Flag vector with AKT-1 insert was purchased from Vigene Biosciences (Jinan, Shandong, China) and used to transiently overexpress AKT-1. The plasmid was transfected into HGC-27 and AGS cells using lipofectamine 3000 (Life Technologies). A total of $24 \mathrm{~h}$ after transfection, AKT-1 expression levels in HGC-27 and AGS cells were confirmed by western blot, and transfected cells were used for subsequent experiments.

\section{Real-time cell impedance analysis}

The xCELLigence system (Roche Applied Science, Mannheim, Germany) was used to dynamically monitor cell proliferation rates. Experiments were performed using a standard protocol developed by Roche Applied Science. Briefly, HGC-27 and AGS cells were seeded into $100 \mu \mathrm{l}$ of media in an E-Plate. Cell proliferation was monitored by 
measuring electrical impedance across microelectrodes on the bottom of the E-Plate. Impedance was expressed as the normalized cell index, which is an arbitrary unit. The results were analyzed using the real-time cell analysis software supplied by the company.

\section{Cell viability assay}

A cell counting kit-8 (CCK-8) assay (Nanjing KeyGen Biotech Co., Ltd.) was used to analyze the effect of chaetocin on GC cell viability. Briefly, $100 \mu \mathrm{l}$ of $1 \times 10^{5} / \mathrm{ml}$ cells were treated with various doses of chaetocin for $24 \mathrm{~h}$. Then, $20 \mu \mathrm{l}$ CCK- 8 reagents were added to each well and incubated for an additional $4 \mathrm{~h}$. The absorbance was measured at $450 \mathrm{~nm}$ using a Varioskan Flash multimode reader (Thermo Fisher Scientific, Waltham, MA, USA).

\section{Colony formation assay}

HGC-27 and AGS cells were seeded in a 6-well plate (500 cells/well) and treated with various concentration of chaetocin. Cells were cultured in a $5 \% \mathrm{CO}_{2}$ atmosphere at $37^{\circ} \mathrm{C}$ for $9 \mathrm{~d}$, and the culture medium was changed every $3 \mathrm{~d}$. After chaetocin treatment, cells were washed with PBS, fixed in ice-cold methanol, and stained with crystal violet. An Epson scanner (Suwa, Nagano, Japan) was used to image the colonies.

\section{Cell cycle analysis by flow cytometry}

After chaetocin treatment, HGC-27 and AGS cells were collected, washed and fixed with cold 66\% ethanol overnight at $4{ }^{\circ} \mathrm{C}$. Cells were then washed in PBS and labeled with $500 \mu \mathrm{l}$ propidium iodide (PI) (BD Biosciences, Franklin Lakes, NJ, USA). The FACSCanto II flow cytometry (BD Bioscience) was used to analyze cell cycle distribution.

\section{Cell apoptosis analysis by flow cytometry}

An annexin V-FITC/PI staining kit (Nanjing KeyGen Biotech Co., Ltd.) or an annexin V-APC/7-aminoactinomycin D (7-AAD) staining kit (MultiSciences (Lianke) Biotech Co., Ltd., Hangzhou, Zhejiang, China) was used to evaluate apoptosis. Following chaetocin treatment, cells were collected, washed and stained in working solution (500 $\mu \mathrm{l}$ binding buffer with $5 \mu \mathrm{l}$ annexin V-FITC and $5 \mu \mathrm{l}$ PI or $500 \mu \mathrm{l}$ binding buffer with $5 \mu \mathrm{l}$ annexin $\mathrm{V}$ - APC and $10 \mu \mathrm{l}$ 7-AAD) for $15 \mathrm{~min}$ at room temperature in the dark. The FACSCanto II flow cytometry (BD Bioscience) was used to detect apoptotic cells. Annexin V-FITC ${ }^{+}$and annexin VFITC $^{+}-\mathrm{PI}^{+}$cells or annexin $\mathrm{V}-\mathrm{APC}^{+}$and annexin $\mathrm{V}$ $\mathrm{APC}^{+} 7-\mathrm{AAD}^{+}$cells were considered apoptotic cells.

\section{Western blot analysis}

Following chaetocin treatment, HGC-27 and AGS cells were lysed in RIPA buffer (Cell Signaling Technology) containing protease and phosphatase inhibitors (Nanjing
KeyGen Biotech Co., Ltd.). The concentration of each protein sample was measured using the Pierce BCA protein assay kit (Thermo Fisher Scientific, Waltham, MA, USA). Total cellular proteins were separated by SDSPAGE and then transferred to PVDF membranes. After blocking in $5 \%$ non-fat dry milk, membranes were probed with primary antibodies overnight at $4{ }^{\circ} \mathrm{C}$. The next day, membranes were washed with PBST, incubated with a horseradish peroxidase-conjugated secondary antibody and finally detected using enhanced chemiluminescence.

\section{TRXR activity assay}

TRXR activity was detected using the TRXR assay kit (Abcam, Cambridge, MA, USA) according to the manufacturer's protocol. Briefly, after chaetocin treatment, HGC-27 and AGS cells were lysed in assay buffer (provided with the kit), and the concentration of each protein sample was determined using the Pierce BCA protein assay kit (Thermo Fisher Scientific). A total of $100 \mu \mathrm{g}$ of each protein sample was mixed with DTNB and NADPH, and the TRXR activity was calculated as the increase in absorbance of $\mathrm{TNB}^{2-}$ at $412 \mathrm{~nm}$ from $1 \mathrm{~min}$ to $20 \mathrm{~min}$. The TRXR activity is shown as \% of control.

\section{Determination of reduced and oxidized forms of TRX-1 in GC cells}

Reduced and oxidized forms of TRX-1 in GC cells were determined as previously described ${ }^{26}$. After chaetocin treatment, HGC-27 and AGS cell lysates were denatured and precipitated by trichloroacetic acid at a final concentration of $7.5 \%$ and centrifuged at $12,000 \mathrm{~g}$ for $10 \mathrm{~min}$ at $4{ }^{\circ} \mathrm{C}$ to collect protein precipitates. After washed with acetone twice, the protein precipitates were solubilized in a buffer containing $50 \mathrm{mM}$ Tris- $\mathrm{HCl}(\mathrm{pH} 7.4), 1 \%$ sodium dodecyl sulfate, and $15 \mathrm{mM}$ 4-acetamido-4'-maleimidylstilbene-2,2'-disulfonic acid (AMS). The reduced and oxidized forms of TRX-1 was separated by non-reducing SDS-PAGE followed by western blot analysis.

\section{Molecular docking simulation}

The docking interaction between chaetocin and TRXR1 was simulated using the AutoDock 4.2. The human TRXR-1 crystal structure (PDB code: $2 Z Z C$ ), which has a resolution of $2.6 \AA$, was retrieved from the RCSB protein data bank and used for docking studies. The structure of chaetocin was assembled using Chemoffice. The binding location was defined on the active binding site of TRXR$1^{27}$, and the grid box (Center: -37.844, 16.961, $-41.324 \AA$ ) was set to $20 \times 22 \times 20 \AA$ in the $\mathrm{X}, \mathrm{Y}$, and $\mathrm{Z}$ directions, with spacing within a $9 \AA$ radius.

\section{Molecular dynamics (MD) simulation}

The complex of TRXR-1 protein and chaetocin obtained from the docking simulation were used as the 
initial coordinates for MD simulations. GROMACS 5.1.4 was used for MD. TRXR-1 and chaetocin were assessed using ff99sb force field and general amber force field, respectively. A truncated octahedron box of transferable intermolecular potential for water molecules with a margin distance of $10 \AA$, periodic boundary conditions and neutralizing counterions was added to the system. The production MD simulation was performed for $15 \mathrm{~ns}$ using the NPT ensemble at a temperature of $300 \mathrm{~K}$, and coordinates were saved every $1 \mathrm{ps}$. The binding free energies of the chaetocin-TRXR-1 interaction were calculated using the molecular mechanics/poisson-boltzmann surface area (MM/PBSA) method.

\section{RNA sequencing (RNA-seq)}

RNA was sequenced and analyzed by Huada (Wuhan, Hubei, China). Briefly, RNA was extracted from nontreated AGS cells as well as AGS cells with $100 \mathrm{nM}$ chaetocin treatment for $12 \mathrm{~h}$. RNA-seq was performed on the BGISEQ-500 platform. Reads were aligned to the human RefSeq hg38 reference genome using Bowtie2. Differentially expressed genes (DEGs) were calculated by RNA-seq by expectation maximization and possion distribution and DEGs defined as $+/-$ twofold change and false discovery rate (FDR) $<0.01$ were analyzed for enriched gene pathways using KEGG pathway analysis (http:// www.genome.jp/kegg/pathway.html). $q$ value of the pathway shown in the figure was $<0.05$.

\section{Determination of cellular oxygen species (ROS)}

Cellular ROS production was measured using a ROS assay kit (Beyotime, Shanghai, China) according to the manufacturer's protocol. Briefly, a total of $1.5 \times 10^{5} / \mathrm{ml}$ cells in $2 \mathrm{ml}$ was seeded in 6-well dishes. Cells were incubated with $10 \mu \mathrm{M} \mathrm{2} 2^{\prime}, 7^{\prime}$-dichlorofluorescin diacetate (DCFH-DA) for $20 \mathrm{~min}$ in the dark and then treated with chaetocin. Cells were then washed with culture media (without fetal bovine serum) and DCFH-DA florescence was measured at $525 \mathrm{~nm}$ using a FACSCanto II flow cytometry (BD Bioscience).

\section{Immunohistochemistry}

Xenograft tumors were fixed in a $4 \%$ formaldehyde solution in PBS, embedded in paraffin and sectioned. Following deparaffinization in xylene and hydration with decreasing concentrations of alcohol, sections were incubated with $0.3 \%$ hydrogen peroxide to block endogenous peroxidase activity and boiled in EDTA buffer $(\mathrm{pH}=8.0)$ for antigen retrieval. Sections were then incubated with a mouse monoclonal ki-67 antibody at $4{ }^{\circ} \mathrm{C}$ overnight in a moist chamber. The next day, sections were washed with PBS, incubated with a horseradish peroxidase-conjugated secondary antibody and finally detected using 3,3'-diaminobenzidine.

\section{Animal model}

We used five-week-old male BALB/c nude mice bred at Experimental Animal Center of Guangdong Laboratory Animals Monitoring Institute for HGC-27 xenograft model. A total of $1 \times 10^{7}$ HGC-27 cells were subcutaneously injected into the flanks of nude mice. When the average tumor volume reached $\sim 50 \mathrm{~mm}^{3}$, mice were divided randomly into two groups (six mice per group) and treated with either vehicle (10\% DMSO, 20\% cremophor $\mathrm{EL}$ and $70 \% \mathrm{NaCl}$, i.p.) or chaetocin $(0.5 \mathrm{mg} / \mathrm{kg}$, i.p.) every other day for a total of $24 \mathrm{~d}$. Tumors were measured every other day, and tumor volumes were calculated using the following formula: $a^{2} \times b / 2$, where $a$ is the smallest diameter and $b$ is the diameter perpendicular to $a$. At the end of the study, tumors were removed from sacrificed mice and then weighed and stored.

The patient-derived xenograft (PDX) models used in this study were established by our group as described previously $^{28}$. Briefly, fresh gastric cancer tissues, which were termed as F0, were cut into approximately $3 \times 3 \times$ $3 \mathrm{~mm}^{3}$ pieces and then were subcutaneously injected into the flanks of five-week-old male NODPrkdc ${ }^{\text {em26Cd52 }} \mathrm{Il} 2$ rg $^{\text {em26Cd22 }} / \mathrm{Nju}$ (NCG) mice (F1). After tumors reached a size of $\sim 500 \mathrm{~mm}^{3}$, they were harvested and passaged into the next generation. One of the successfully established PDX models in the third generation (F3) was used in this study. After $19 \mathrm{~d}$ of inoculation, mice were divided randomly into two groups (five mice per group) and treated with either vehicle (10\% DMSO, 20\% cremophor $\mathrm{EL}$ and $70 \% \mathrm{NaCl}$, i.p.) or chaetocin $(0.25 \mathrm{mg} /$ $\mathrm{kg}$, i.p.) every other day for a total of $14 \mathrm{~d}$. Tumors were measured every other day, and tumor volumes were calculated using the following formula: $\mathrm{a}^{2} \times \mathrm{b} / 2$, where $\mathrm{a}$ is the smallest diameter and $b$ is the diameter perpendicular to a. At the end of the study, tumors were removed from sacrificed mice and then weighed and stored.

All animal studies were conducted according to the guidelines of Animal Care and Use Committee of Guangdong Laboratory Animals Monitoring Institute.

\section{Statistical analysis}

All experiments were performed at least 3 times, and the results are presented as the mean \pm SD where applicable. Statistical analysis was performed using oneway analysis of variance followed by tukey's test among multiple groups and Student's $t$-test between two groups by GraphPad Prism software (San Diego, CA, USA). $P<$ 0.05 was considered statistically significant.

\section{Results \\ Chaetocin inhibits the growth of GC cells}

The chemical structure of chaetocin is shown in Fig. 1a. We first investigated the cytotoxic effects of chaetocin in GC cells and gastric mucosa epithelial cell line GES-1. 


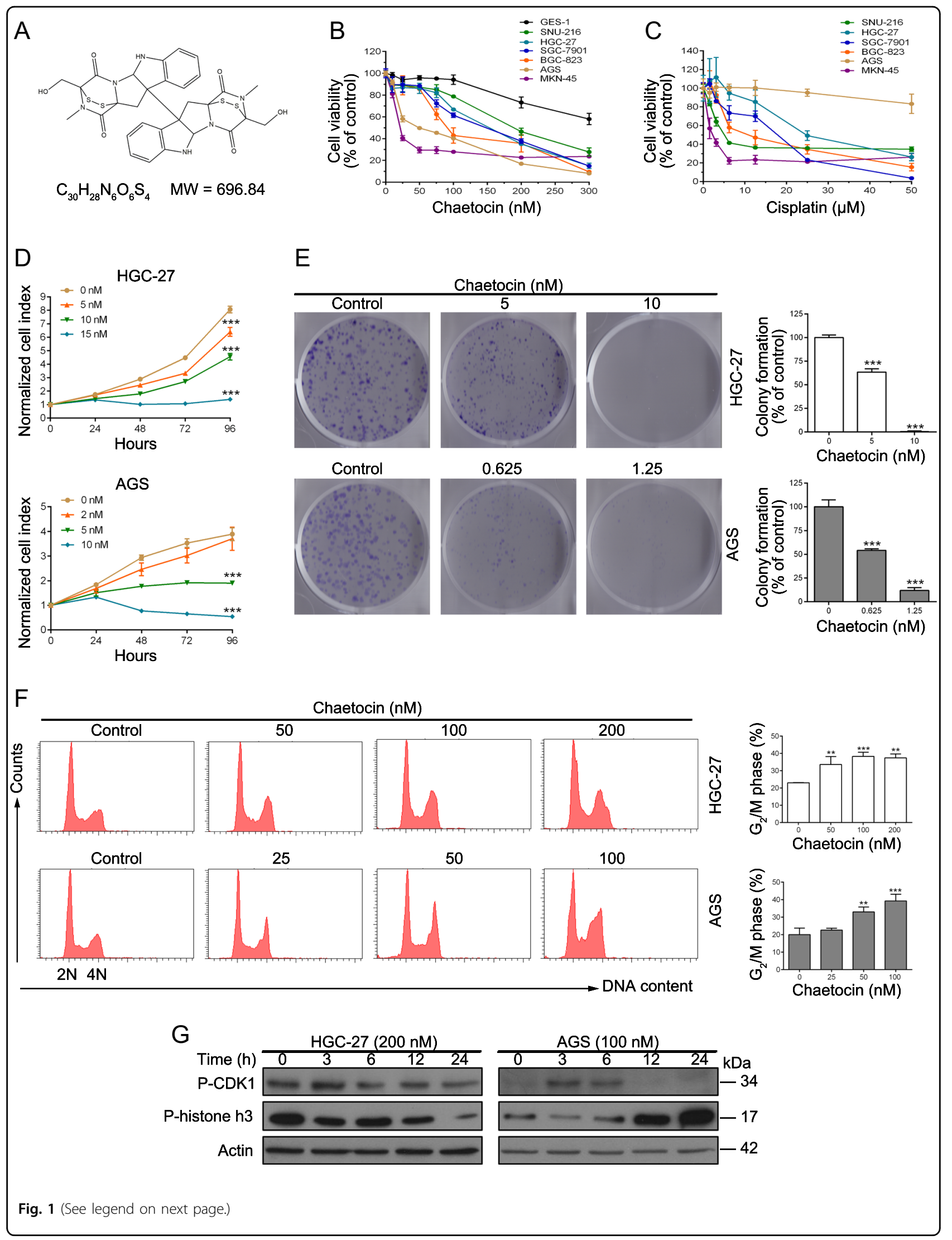


(see figure on previous page)

Fig. 1 Chaetocin induces $G_{2} / M$ phase arrest and inhibits proliferation in GC cells. a Chemical structure of chaetocin. $\mathbf{b}$ and $\mathbf{c} C C K-8$ assays were used to measure cell viability following treatment with chaetocin or cisplatin at the indicated concentrations for $24 \mathrm{~h}$. $\mathbf{d}$ HGC-27 and AGS cells were treated with various concentrations of chaetocin and then cell proliferation was detected by real-time analysis. e HGC-27 and AGS cells were incubated with the indicated concentrations of chaetocin for $9 \mathrm{~d}$ and then HGC-27 and AGS cell colonies were counted. $\mathbf{f}$ HGC-27 and AGS cells were exposed to the indicated concentrations of chaetocin for $12 \mathrm{~h}$ and then cell cycle distribution was analyzed by flow cytometry. $\mathbf{g}$ Expression levels of p-CDK1 and p-histone h3 in HGC-27 and AGS were detected by western blot analysis. Blots presented here are representative of three independent experiments. Results in $(\mathbf{b})-(\mathbf{f})$ are shown as mean \pm SD of three independent experiments. ${ }^{* *} P<0.01,{ }^{* * *} P<0.001$, vs. control group

Cells were treated with chaetocin for $24 \mathrm{~h}$, and cell viability was detected using a CCK-8 assay. As shown in Fig. $1 \mathrm{~b}$ and Table $1, \mathrm{GC}$ cell viability was significantly inhibited by very low concentrations of chaetocin $\left(\mathrm{IC}_{50}\right.$ values between 185.7 and $31.0 \mathrm{nM}$ ) and GC cells seemed to be more sensitive to chaetocin compared with GES-1. Surprisingly, $\mathrm{IC}_{50}$ values of chaetocin to $\mathrm{GC}$ cells were lower to those of cisplatin (Fig. 1c and Table 2), which is one of the most effective chemotherapeutic agents used for GC treatment. Two cell lines HGC-27 and AGS, which showed different sensitive to chaetocin, were chosen for further study. To test the effect of chaetocin on GC cell proliferation, real-time cell analysis and colony formation assays were then performed and low concentrations which wouldn't cause cell death were chosen. Chaetocin reduced both the normalized cell index and colony formation in a dose-dependent manner in HGC-27 and AGS cells (Fig. 1d, e), indicating that GC cell proliferation was markedly restrained. In addition, we used flow cytometry to evaluate the effect of chaetocin on cell cycle distribution. As shown in Fig. 1f, after $12 \mathrm{~h}$ of chaetocin treatment, the population of HGC-27 and AGS cells in the $\mathrm{G}_{2} / \mathrm{M}$ phase increased substantially, suggesting that chaetocin induces $\mathrm{GC}$ cell arrest in the $\mathrm{G}_{2} / \mathrm{M}$ phase. To determine $\mathrm{GC}$ cells were arrested in which phases: $\mathrm{G}_{2}$ or mitosis, the expression of $\mathrm{p}$-CDK1 and p-histone $\mathrm{h} 3$ proteins were tested; the expression levels of both $\mathrm{p}$ CDK1 and p-histone h3 decrease if cells are arrested in $\mathrm{G}_{2}$ phase and the expression levels of p-histone $\mathrm{h} 3$ increase during mitosis ${ }^{29,30}$. Interestingly, the expressions of both $\mathrm{p}-\mathrm{CDK} 1$ and p-histone h3 decreased upon chaetocin treatment in HGC-27 cells while the expression of phistone h3 increased in AGS cells (Fig. 1g), indicating that chaetocin causes $\mathrm{G}_{2}$ arrest in HGC-27 cells while mitotic arrest in AGS cells.

\section{Chaetocin induces caspase-dependent apoptosis in GC cells}

We next determined whether chaetocin could induce apoptotic cell death in GC cells. HGC-27 and AGS cells were exposed to chaetocin and then assessed by flow cytometry with annexin V-FITC/PI staining. Apoptotic HGC-27 and AGS cell populations increased significantly following chaetocin treatment (Fig. 2a). To further
Table 1 Effects on viability of chaetocin to GC cells and GES-1

\begin{tabular}{ll}
\hline Cell line & IC $_{\mathbf{5 0}}(\mathbf{n M})$ \\
\hline GES-1 & $>300$ \\
SNU-216 & $185.7 \pm 13.9$ \\
HGC-27 & $141.80 \pm 19.6$ \\
SGC-7901 & $133.9 \pm 12.8$ \\
BGC-823 & $104.4 \pm 4.3$ \\
AGS & $51.7 \pm 7.8$ \\
MKN-45 & $31.0 \pm 5.6$ \\
\hline
\end{tabular}

Table 2 Effects on viability of cisplatin to various GC cells

\begin{tabular}{ll}
\hline Cell line & IC $_{\mathbf{5 0}}(\boldsymbol{\mu M})$ \\
\hline SNU-216 & $7.9 \pm 0.6$ \\
HGC-27 & $27.0 \pm 3.0$ \\
SGC-7901 & $15.1 \pm 3.1$ \\
BGC-823 & $12.2 \pm 2.9$ \\
AGS & $>50$ \\
MKN-45 & $1.5 \pm 0.5$ \\
\hline
\end{tabular}

confirm the chaetocin-induced apoptotic effect, apoptosis-related proteins were detected by western blot assay. As shown in Fig. 2b, chaetocin-induced PARP cleavage (an indicator of apoptosis) in HGC-27 and AGS cells. Further, caspase family proteins (known upstream activators of PARP) were activated. Chaetocin decreased levels of the precursor forms of caspase- $3,-9$, and -8 and increased the cleaved forms of caspase- $3,-9$, and -8 in HGC-27 and AGS cells, illustrating that chaetocin may induce caspase-dependent apoptosis of GC cells. Finally, we measured the expression of BCL-2 and IAP family proteins, as these proteins can affect apoptosis rates. Western blot analysis demonstrated that levels of the antiapoptotic proteins BCL-2, BCL-XL, MCL-1, XIAP and survivin decreased following chaetocin treatment (Fig. 2c). Taken together, these results showed that chaetocin induces GC cell apoptosis. To verify whether the 


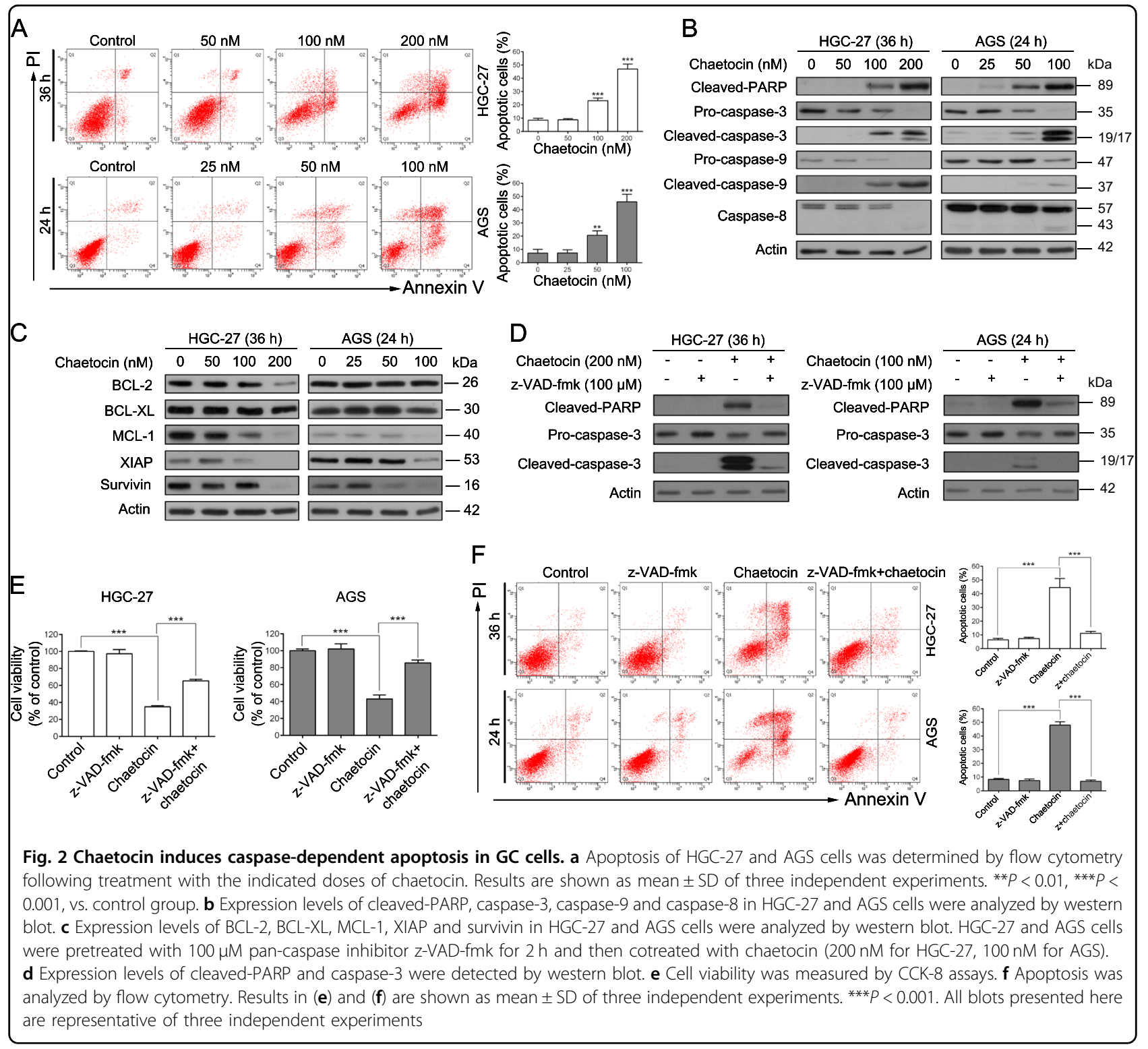

chaetocin-induced GC cell apoptosis was caspase-dependent, we treated cells with the pan-caspase inhibitor $\mathrm{z}$ VAD-fmk and then assessed chaetocin-induced apoptotic effects. z-VAD-fmk significantly blocked chaetocininduced caspase pathway activation (Fig. 2d). Figure 2e, $\mathrm{f}$ showed that chaetocin-induced viability inhibition and apoptotic effect was significantly reversed by z-VAD-fmk treatment in HGC-27 and AGS cells, indicating that chaetocin induces apoptosis in GC cells through the caspase pathway.

\section{Chaetocin induces GC cell apoptosis by inhibiting TRXR-1 activity}

As the TRXR-1-inhibitory effects of chaetocin have already been described in cell-free assays ${ }^{25}$, we first performed molecular docking studies to further confirm the chaetocin-TRXR-1 interaction and to investigate possible binding sites. The docking results showed that chaetocin could bind TRXR-1 directly with a binding energy as high as $-9.5 \mathrm{kcal} / \mathrm{mol}$. The binding interactions consisted of a hydrogen bond between chaetocin and either the Tyr116 or the Glu477 residue in TRXR-1 (Fig. $3 a)$. As the molecular docking method indicated a possibly instantaneous binding mode, we then used MD simulations to evaluate the reasonable/stable binding patterns between chaetocin and TRXR-1. As shown in Fig. 3b, the root mean square deviation (RMSD) curve was stable at approximately $1.8-2.2 \AA$ after $2 \mathrm{~ns}$, implying a powerful binding interaction between chaetocin and TRXR-1. To calculate the binding free energy and speculate upon the 


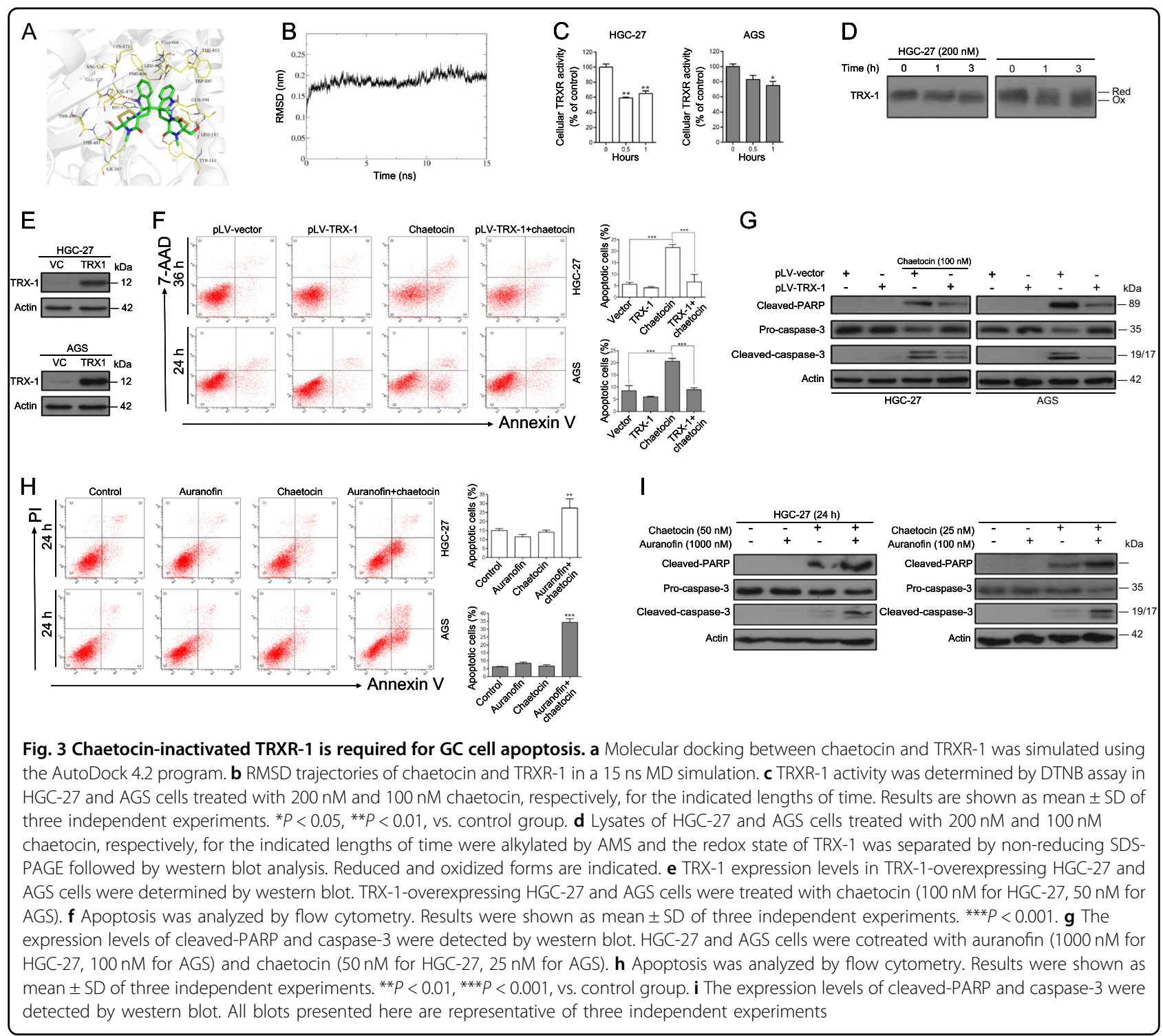

possible residues involved in chaetocin binding, we used MM/PBSA to investigate the detailed changes in 200 frames within the final $5 \mathrm{~ns}$. The binding free energy between chaetocin and TRXR-1 is $-157.013 \mathrm{~kJ} / \mathrm{mol}$ and the top 10 possible binding residues are shown in Table 3 . Consistent with the docking results, chaetocin can form two hydrogen bonds with Glu477. We next tested the inhibitory effects of chaetocin on TRXR-1 activity in GC cells. Following treatment of HGC-27 and AGS cells with chaetocin, the activity of TRXR-1 was significantly inhibited (Fig. 3c). And this effect is similar to auranofin, which has been previously reported to induce GC cell death by inhibiting the activity of TRXR-1 followed by inducing ROS accumulation ${ }^{31,32}$ (Supplementary Figs. 13 , Supplementary Table 1). TRXR-1 provides reducing equivalents to the downstream effector TRX-1, which is ultimately responsible for the antioxidant effects ${ }^{5}$. The inhibition of TRXR-1 activity induced by chaetocin might lead to a decrease of reduced TRX-1. We then investigated the redox state of TRX-1 in GC cells by using AMS alkylation method. As shown in Fig. 3d, the amount of oxidized TRX-1 increased when GC cells were treated with chaetocin. Taken together, these results demonstrate that chaetocin can inactivate TRXR-1 in GC cells.

As chaetocin-induced inhibition of TRXR-1 may result in a reduction of reduced TRX-1, and finally induce cell apoptosis via accumulation of ROS. We therefore hypothesized that increased TRX-1 could diminish chaetocininduced apoptosis. We constructed cells that stably overexpressed TRX-1 (Fig. 3e) and found that stable TRX1 overexpression in HGC-27 and AGS cells attenuated chaetocin-induced apoptosis (Fig. 3f, g). We next investigated whether chaetocin-induced apoptosis was increased when cells were cotreated with auranofin, a 
Table 3 Top 10 possible binding residues in TRXR-1 with chaetocin

\begin{tabular}{lc}
\hline Residues & MM energy (kJ/mol) \\
\hline Glu-477 & -33.69 \\
Gln-494 & -21.56 \\
Thr-481 & -7.78 \\
Val-478 & -7.68 \\
Tyr-116 & -7.24 \\
Glu-122 & -6.04 \\
Gly-499 & -5.86 \\
Pro-344 & -5.41 \\
His-472 & -5.41 \\
Thr-480 & -4.75 \\
\hline
\end{tabular}

reported inhibitor of thioredoxin reductase. The combined treatment induced much higher apoptosis in GC cells than that of single agent (Fig. 3h, i). These results suggest that chaetocin induces GC cell apoptosis by inhibiting TRXR-1 activity.

\section{Chaetocin-induced GC cell apoptosis depends on ROS generation}

Because the TRX-TRXR system acts as a ROS scavenger, we next determined ROS levels in chaetocintreated GC cells. ROS levels in GC cells, detected using a fluorescent DCFH/DA probe, increased significantly upon chaetocin treatment (Fig. 4a). Previous studies have demonstrated that increased ROS production is crucial for chaetocin-induced apoptotic cell death in leukemia, glioma, and myeloma cells ${ }^{18,33,34}$. Therefore, we next determined the role of ROS in the chaetocin-induced apoptosis of GC cells. We used NAC to inhibit the production of ROS (Fig. 4a). Flow cytometry analysis demonstrated that the chaetocin-induced increase in apoptotic cells was almost completely reversed upon cotreatment with NAC (Fig. 4b). In accordance with the flow cytometric results, western blot demonstrated that NAC treatment could reverse the chaetocin-induced increase in cleaved PARP and caspase family proteins (Fig. 4c). These results suggest that chaetocin induces ROS production by inhibiting the TRX-TRXR system and that this ROS production is required for chaetocinmediated apoptosis in GC cells.

\section{The PI3K/AKT pathway is involved in chaetocin-induced GC cell apoptosis}

To demonstrate the detailed mechanism underlying TRXR-1/TRX-1/ROS-mediated apoptosis, we performed RNA-seq analysis of chaetocin-treated AGS cells. Several pathways were affected by chaetocin treatment; one of the most enriched pathways was the PI3K/AKT pathway (Fig. 5a). The PI3K/AKT pathway is one of the most well-known ROS-regulated pathways and plays an important role in cancer progression by promoting cancer cell proliferation and inhibiting cancer cell apoptosis ${ }^{35,36}$. Thus, we focused on the role of the PI3K/AKT pathway in chaetocin-mediated cell death. We first verified the effect of chaetocin on the PI3K/AKT pathway in HGC-27 and AGS cells. The expression of some genes, like c-Myc, Axin-2, BCL-XL, MCL-1 and XIAP, may be downregulated if the PI3K/ AKT pathway is inactivated ${ }^{37}$. Our results revealed that the mRNA expression levels of c-Myc, Axin-2, BCL-XL, MCL-1, and XIAP were downregulated in GC cells treated with chaetocin (Supplementary Fig. 4), which is consistent with the RNA-seq analysis. Moreover, following chaetocin treatment, phospho-AKT (Ser473) expression decreased in a dose- and time-dependent manner, while total AKT expression remained nearly unchanged (Fig. 5b). These results indicate that the PI3K/AKT pathway is inactivated by chaetocin in GC cells. To further confirm that the PI3K/AKT pathway is involved in chaetocin-induced cell death, we transiently overexpressed AKT-1 in GC cells and then observed the effect of chaetocin on these cells (Fig. 5c). Both flow cytometry and western blot demonstrated that AKT-1 overexpression could slightly attenuate chaetocininduced apoptosis (Fig. 5d, e). Moreover, the PI3K/ AKT pathway inhibitor LY294002 significantly enhanced chaetocin-induced apoptotic effects (Fig. 5f, g). Studies have reported that ROS can repress the PI3K/ AKT pathway ${ }^{38}$. Our results revealed that the ROS scavenger NAC could rescue the chaetocin-induced decrease in p-AKT levels (Fig. 5h). These data indicate that the chaetocin-mediated inactivation of the PI3K/ AKT pathway depends on ROS generation and is involved in chaetocin-induced cell apoptosis.

\section{Chaetocin inhibits the growth of GC cell xenografts and patient-derived GC xenografts}

The effect of chaetocin on GC cells in vivo was examined using a nude mouse xenograft model. HGC-27 cells were subcutaneously implanted into nude mice. Then, mice were treated with either vehicle or chaetocin $(0.5 \mathrm{mg} / \mathrm{kg}$, i.p.) every other day for $24 \mathrm{~d}$. Compared with vehicle treatment, chaetocin effectively reduced the volume and weight of HGC-27 xenograft tumors (Fig. $6 \mathrm{a}-\mathrm{c})$, and no significant losses were detected in the body weights of the experimental animals (Fig. 6d), suggesting that chaetocin significantly restrains the growth of GC cell xenografts but has no major side effects in mice. Consistent with our in vitro results, chaetocin-treated tumor 
A
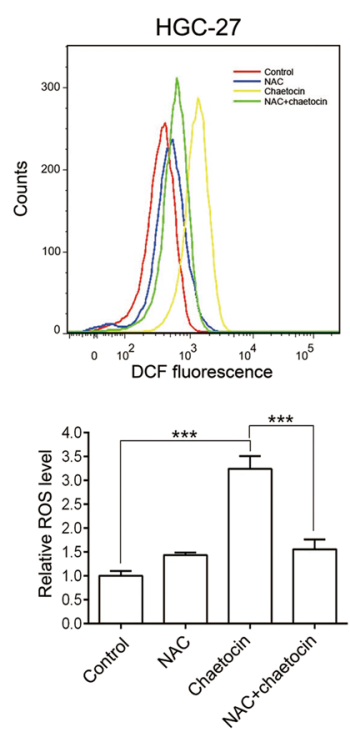

B
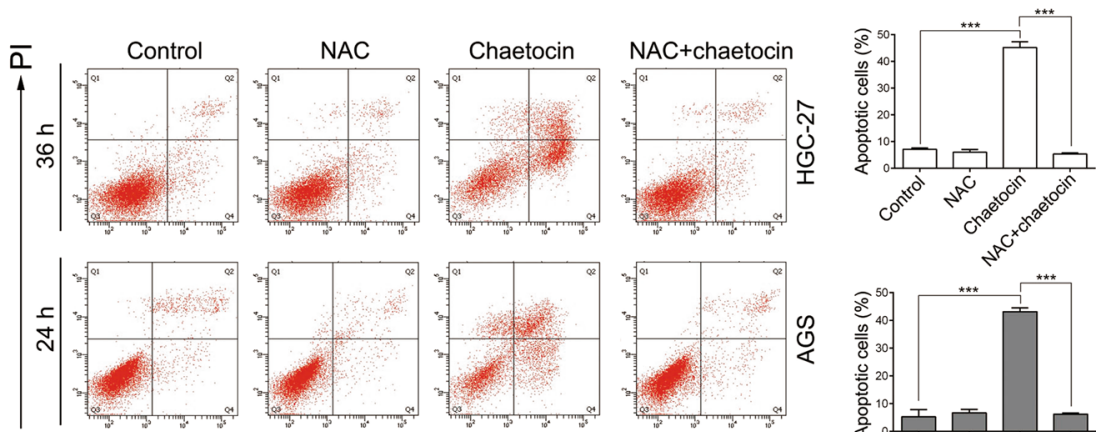

Annexin V
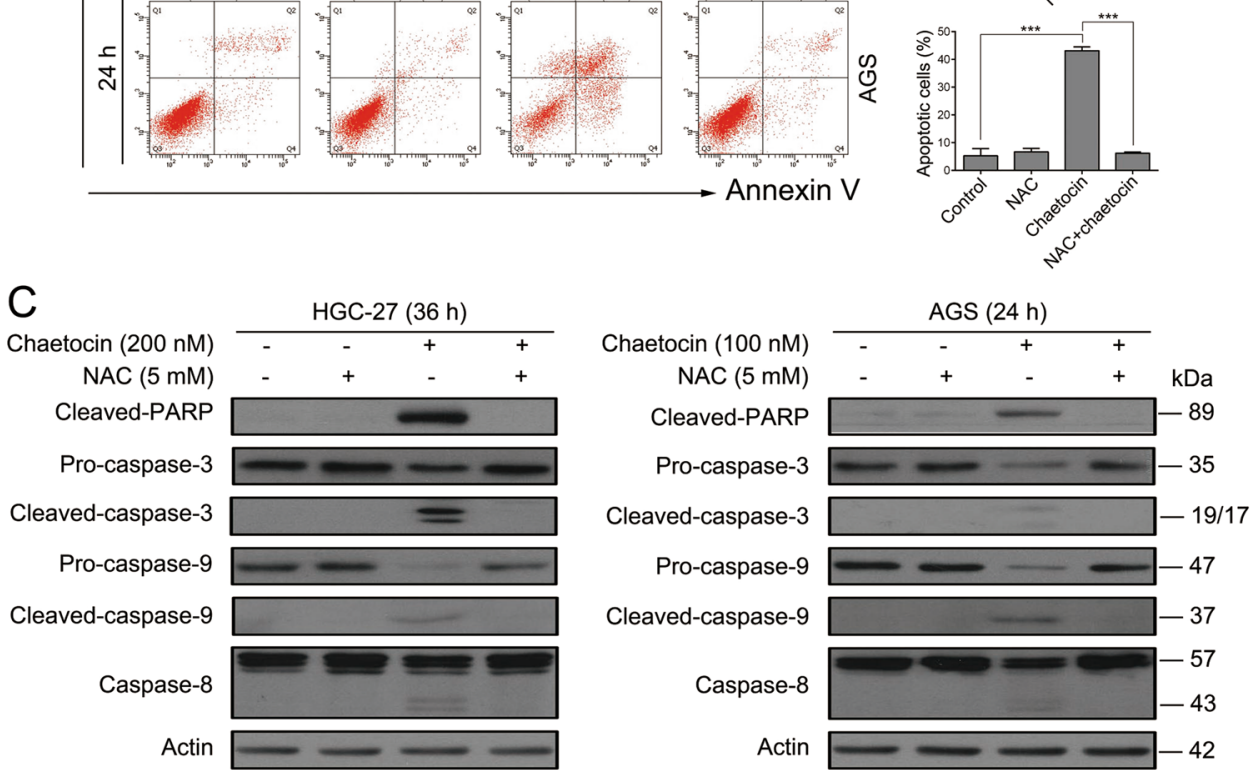

Fig. 4 Chaetocin-induced accumulation of ROS is crucial for GC cell apoptosis. a ROS levels were analyzed by flow cytometry in HGC-27 and AGS cells treated for $1 \mathrm{~h}$ with $5 \mathrm{mM}$ NAC, chaetocin (200 nM for HGC-27, $100 \mathrm{nM}$ for AGS), or both. Results were shown as mean \pm SD of three independent experiments. ${ }^{* * *} P<0.001$. HGC-27 and AGS cells were pretreated with $5 \mathrm{mM} \mathrm{NAC}$ for $1 \mathrm{~h}$ and then cotreated with chaetocin (200 nM for HGC-27, $100 \mathrm{nM}$ for AGS). b Apoptosis was measured by flow cytometry. Results were shown as mean \pm SD of three independent experiments. ${ }^{* * *} P$ $<0.001$. c Expression levels of cleaved-PARP, caspase-3, caspase- 9 and caspase-8 were analyzed by western blot. Blots presented here are representative of three independent experiments

tissues displayed decreased pro-caspase- 3 and p-AKT protein levels and attenuated TRXR activity (Fig. 6e, f). Moreover, immunohistochemistry staining demonstrated that the expression of ki-67, a biomarker related to proliferation, was downregulated in response to chaetocin (Fig. 6g). 


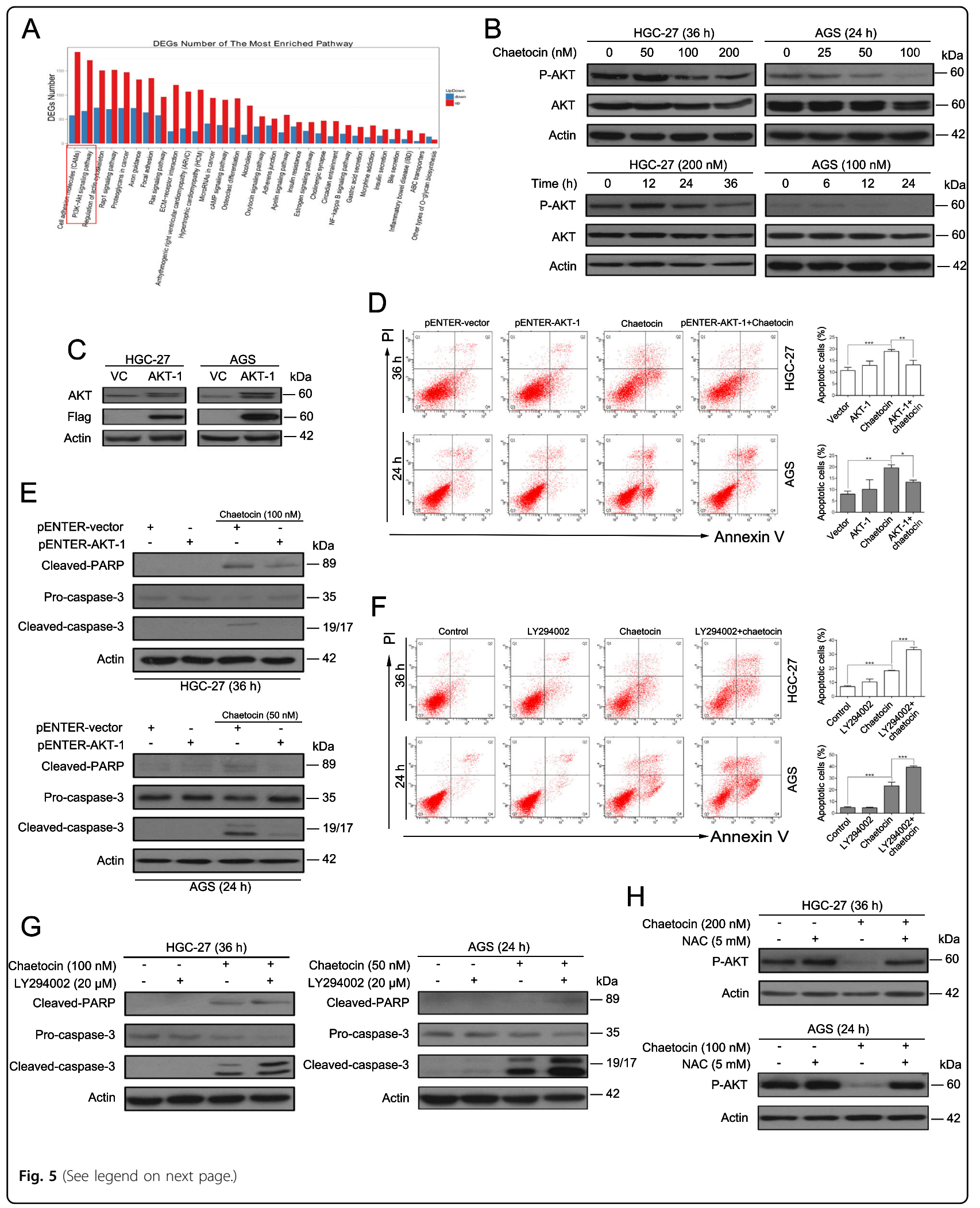


(see figure on previous page)

Fig. 5 The PI3K/AKT pathway is involved in chaetocin-induced apoptosis in GC cells. a RNA-seq was performed on non-treated AGS cells as well as AGS cells with $100 \mathrm{nM}$ chaetocin treatment for $12 \mathrm{~h}$. And DEGs defined as $+/-2$-fold change and FDR $<0.01$ were included for enriched gene pathway analysis. $\mathbf{b}$ p-AKT and AKT expression levels were analyzed by western blot in chaetocin-treated HGC-27 and AGS cells. c AKT and flagtag expression levels were detected by western blot in HGC-27 and AGS cells transiently overexpressing AKT. AKT-overexpressing HGC-27 and AGS cells were treated with chaetocin (100 nM for HGC-27, $50 \mathrm{nM}$ for AGS). d Apoptosis was analyzed by flow cytometry. Results were shown as mean \pm SD of three independent experiments. ${ }^{*} P<0.05,{ }^{* *} P<0.01,{ }^{* * *} P<0.001$. e Cleaved-PARP and caspase-3 expression levels were determined by western blot. HGC-27 and AGS cells were pretreated with $20 \mu \mathrm{M} \mathrm{LY} 294002$ for $2 \mathrm{~h}$ and then cotreated with chaetocin (100 nM for HGC-27, $50 \mathrm{nM}$ for AGS). (f) Apoptosis was analyzed by flow cytometry. Results were shown as mean \pm SD of three independent experiments. ${ }^{* * *} P<0.001$. g CleavedPARP and caspase-3 expression levels were determined by western blot. (h) HGC-27 and AGS cells were pretreated with 5 mM NAC for $1 \mathrm{~h}$ and then cotreated with chaetocin (200 nM for HGC-27, 100 nM for AGS). Then, p-AKT expression levels were analyzed by western blot. All blots presented here are representative of three independent experiments

PDX animal models maintain the heterogeneity of human cancers and can accurately predict the responses of clinical drugs or novel agents in cancer treatment. We then detected the effect of chaetocin on patient-derived $\mathrm{GC}$ xenografts. Aligned with the results from the GC cell xenografts, the volume and weight of PDX were significantly deceased in chaetocin-treated group Compared with the vehicle group and no significant changes in mice body weights were found in both groups (Fig. 7a-d).

Taken together, these data indicate that chaetocin inhibits tumor growth in vivo by targeting TRXR-1.

\section{Discussion}

GC is one of the most common and most lethal cancers worldwide ${ }^{1}$. Although traditional chemotherapeutic drugs such as cisplatin and new molecular targeted agents such as trastuzumab can improve the survival rates of GC patients, the curative effects of these drugs are still not satisfactory ${ }^{2,3}$. Therefore, novel therapeutic agents need to be developed for the treatment of GC. In the present study, we determined that low concentrations of chaetocin could induce growth inhibition and caspasedependent apoptosis in GC cells both in vitro and in vivo through the inhibition of TRXR-1 and the subsequent accumulation of ROS. Moreover, the ROSdependent inactivation of the PI3K/AKT pathway was involved in chaetocin-induced cell apoptosis. To our knowledge, this is the first report to demonstrate that chaetocin inhibits the proliferation and induces apoptosis of GC cells in vitro and in vivo, which suggests that chaetocin may be a potential candidate for GC treatment.

ROS play important roles in cell growth, differentiation and death through the modification of various signaling molecules $^{39-41}$. It has been demonstrated that ROS are implicated in diverse diseases, including cancers. Compared with their normal counterparts, cancer cells display inherently elevated ROS levels. Increased ROS levels are closely related to cancer initiation, metastasis, and drug resistance ${ }^{42,43}$. Although moderate increases in ROS levels can be beneficial to cancer cells, excessive amounts of ROS can cause cell death ${ }^{44,45}$. Therefore, by modulating
ROS levels, redox-targeted agents may effectively kill cancer cells. In fact, some small molecules (for example, cardamonin and furanodienone) have been shown to effectively eliminate cancer cells by increasing ROS levels in cancer cells ${ }^{46,47}$. By upregulating the antioxidant system, cancer cells hinder excessive ROS accumulation and prevent ROS buildup beyond lethal levels. One of the most important antioxidant systems is the TRX-TRXR system $^{48}$. Interestingly, the TRX-TRXR system has been found to be hyperactivated in many cancers including gastric, colorectal and lung cancer. Further, hyperactivation of the TRX-TRXR system plays a critical role in tumorigenesis and cancer progression ${ }^{8-11,49}$. Therefore, the TRX-TRXR system may be a valuable drug target for cancer therapy. Indeed, some agents such as cisplatin ${ }^{50,51}$, one of the most common chemotherapeutic agents used to treat GC, can effectively eliminate cancer cells by inhibiting the TRX-TRXR system. Although cisplatin has been shown to inhibit the viability of GC cells by targeting TRXR-1, the curative effects of cisplatin are still not satisfactory. Therefore, new and more effective agents need to be developed.

Recently, chaetocin, a small-molecule natural product produced by the Chaetomium species of fungi, has been shown to have strong cytotoxic effects against human cancer cells ${ }^{15,19}$. The cytotoxic effects of chaetocin against cancer cells may be related to TRXR-1 inhibition ${ }^{25,34}$. However, the pharmacological mechanism of action of chaetocin in GC has not yet been reported. Our results showed that very low concentrations of chaetocin can inhibit growth and induce apoptotic cell death in GC cells (Figs. 1, 2). We further demonstrated that chaetocin increased cellular ROS production via inhibition of TRXR-1 in GC cells (Figs. 3c and 4a). Moreover, our results showed that overexpression of TRX-1 or cotreatment with NAC could attenuate the cytotoxic effects of chaetocin in GC cells (Figs. 3f, g and 4b, c). These results present convincing evidence that chaetocin is an effective antiGC agent and that its effect may depend on the inhibition of TRXR- 1 and the subsequent accumulation of excessive ROS levels. 
A

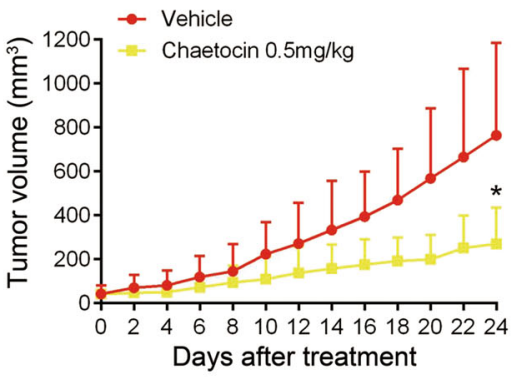

B

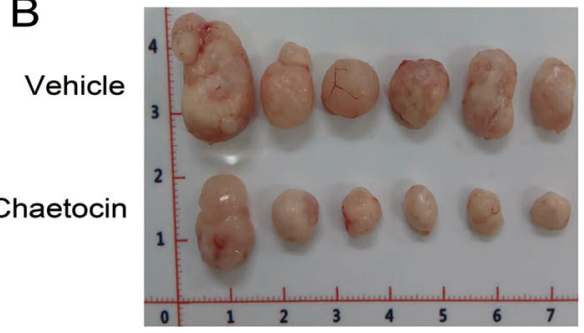

C

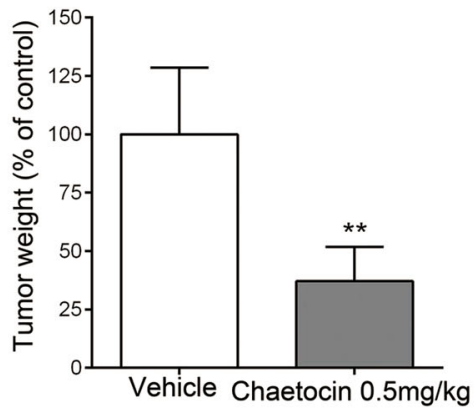

D

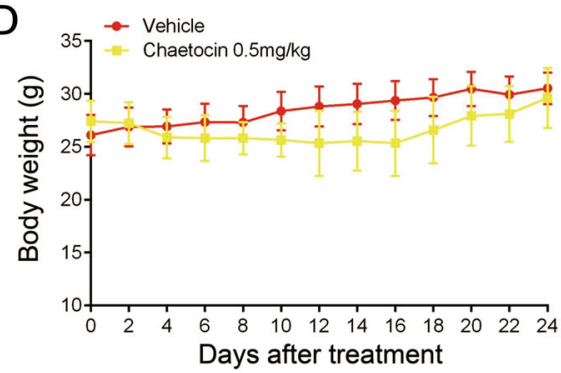

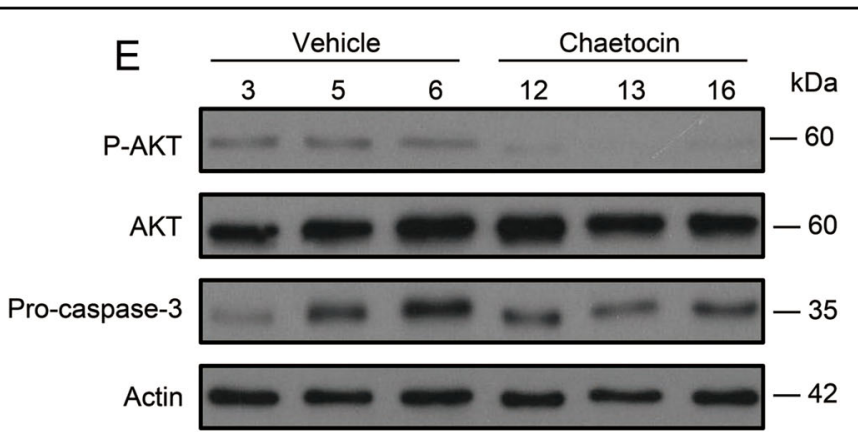

F

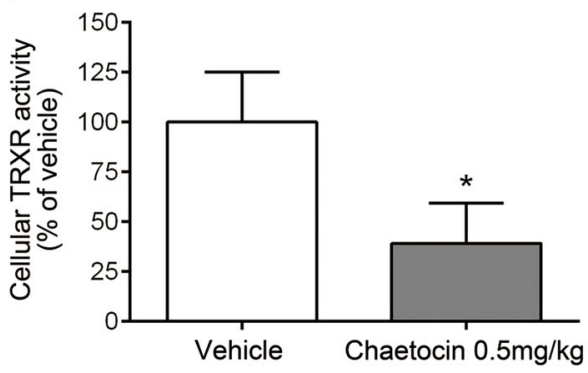

G
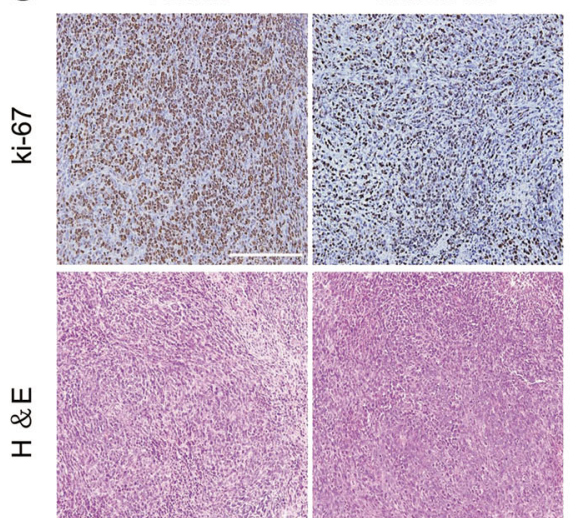

Fig. 6 Chaetocin inhibits the growth of HGC-27 cell xenografts. Nude mice were subcutaneously inoculated with HGC-27 and then treated with vehicle or chaetocin $(0.5 \mathrm{mg} / \mathrm{kg})$ for $24 \mathrm{~d}$. $\mathbf{a}$ and $\mathbf{b}$ tumor volume, and $\mathbf{c}$ tumor weight of HGC-27 GC cell xenografts in nude mice. $\mathbf{d}$ Body weight of the mice. e Levels of p-AKT, AKT and pro-caspase-3 in tumor tissues were detected by western blot (HGC-27 vehicle group: 3, 5, 6; HGC-27 chaetocintreated group: 12, 13, 16). f TRXR activity in tumor tissues was determined by DTNB assay. $\mathbf{g}$ Ki-67 expression in tumor tissues was detected using immunohistochemistry. Scale bar: $100 \mu \mathrm{m}$. Results were shown as mean \pm SD of six mice in each group. ${ }^{*} P<0.05,{ }^{* *} P<0.01, \mathrm{vs}$. control group

Human TRXR-1 contains two identical subunits arranged head-to-tail. A Cys59-Cys64 active site pair in one subunit receives electrons from NADPH and transfers them to the Cys497-selenoCys498 pair at the Cterminus of the other subunit; in this manner, TRXR-1 is maintained in its reduced form and can function as an active enzyme ${ }^{52,53}$. Tibodeau et al. found that chaetocicn inhibited the activity of TRXR-1 in the cell-free system and postulated that chaetocin might interact with the Cys497-selenoCys498 pair at the C-terminus according to the above theories ${ }^{25}$. Our results showed that chaetocin binds to TRXR-1 and inhibits its activity in GC cells. However, our molecular docking and MD simulation results indicated that chaetocin primarily interacts with 


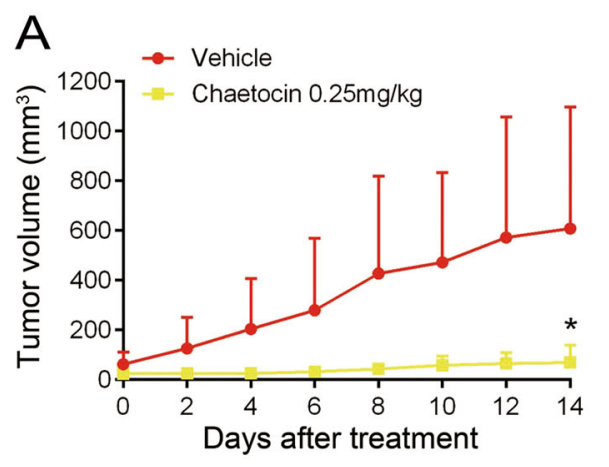

B
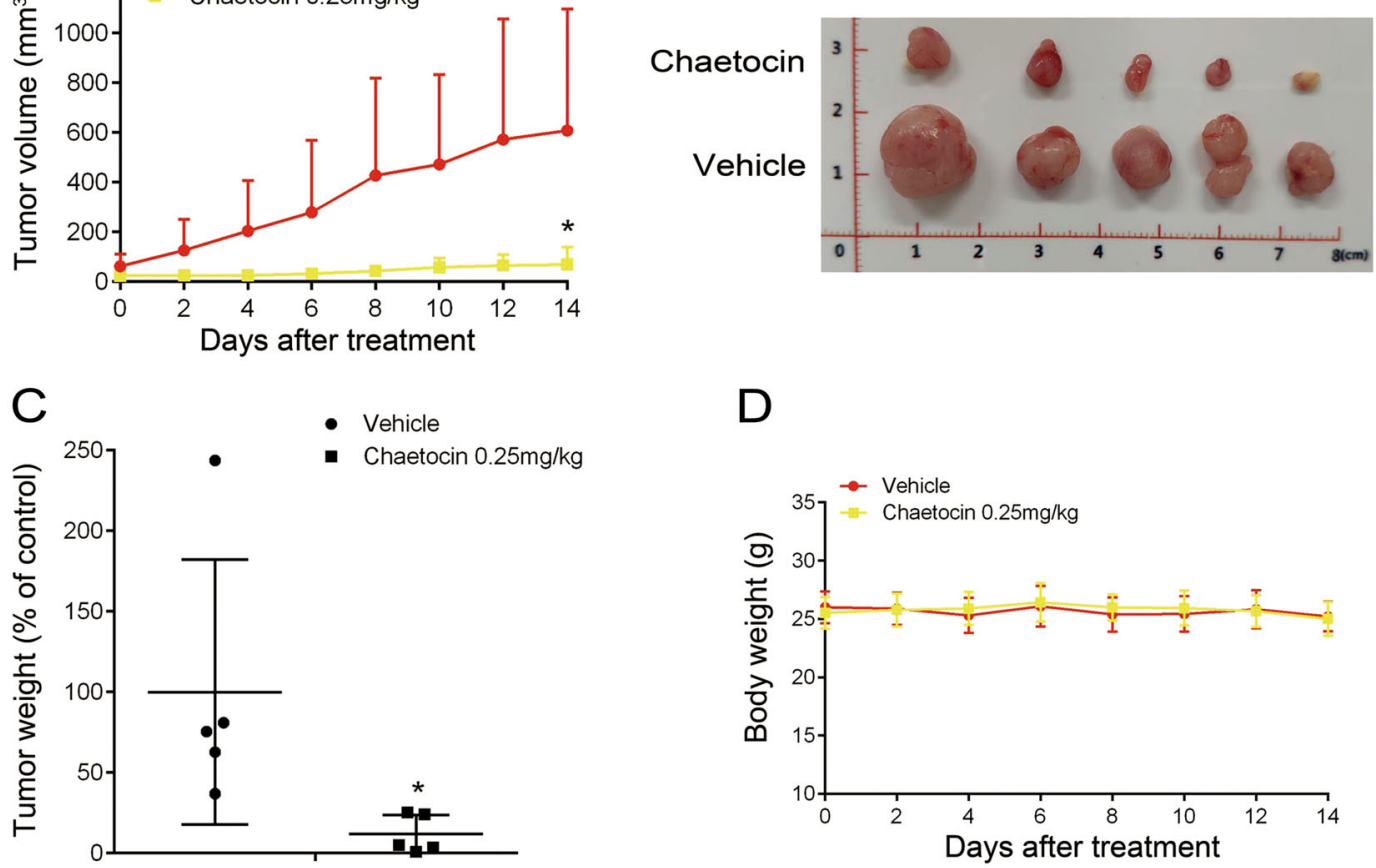

Fig. 7 Chaetocin inhibits the growth of patient-derived GC xenografts. NCG mice were subcutaneously inoculated with GC tissues and then treated with vehicle or chaetocin $(0.25 \mathrm{mg} / \mathrm{kg})$ for $14 \mathrm{~d}$. $\mathbf{a}$ and $\mathbf{b}$ tumor volume, and $\mathbf{c}$ tumor weight of patient-derived xenografts in NCG mice. d Body weight of the mice. Results were shown as mean \pm SD of five mice in each group. ${ }^{*} P<0.05$, vs. control group

the Glu-477 residue through the formation of hydrogen bonds (Fig. 3a, b). As the structure of TRXR-1 in cells changes dynamically, our simulation results may not accurately depict the final binding mode between chaetocin and the active pocket of TRXR-1. Therefore, in the future, bioengineering techniques should be utilized to determine the exact binding sites of chaetocin with TRXR-1 in GC cells.

It has been reported that chaetocin may exhibit its anticancer activity by interacting with other targets: lysine 9 on histone H3 methyltransferase SUV39H1and HIF$1 \alpha^{23,24}$. Some recent findings have demonstrated that chaetocin is a nonspecific inhibitor of SUV $39 \mathrm{H} 1^{54}$. Further, our results showed that the ROS scavenger NAC partly rescued chaetocin-induced inhibition of trimethylation of lysine 9 on histone H3 (H3K9me3), indicating that SUV39H1 may be one of the downstream molecules involved in the ROS pathway (Supplementary Fig. 5) ${ }^{33,34}$. Isham et al. found that the anticancer effects of chaetocin did not depend on functional HIF- $1 \alpha^{19}$. Of note, the anticancer mechanism of chaetocin may vary across different cancers. And the ROS scavenger NAC was able to nearly completely abrogate the anticancer activity of chaetocin in GC cells (Fig. 4b, c), implying that the cytotoxic effects of chaetocin in GC cells may be primarily due to its inhibition of TRXR-1 and the subsequent excessive ROS accumulation, at least in our in vitro model system.

Deregulation of the PI3K/AKT pathway has been reported in various types of cancers, including GC. Additionally, the PI3K/AKT pathway plays an important role in cancer cell proliferation, metastasis, and drug resistance $^{36}$. It is widely known that the PI3K/AKT pathway is a terrific potential molecular target for cancer therapies. Previous studies have shown that ROS can repress the PI3K/AKT pathway ${ }^{38,55}$. However, the relationship between ROS and the PI3K/AKT pathway following chaetocin treatment has not been illustrated yet. Using RNA-seq analysis, we found that the PI3K/AKT pathway is one of the most enriched pathways in chaetocin-treated GC cells (Fig. 5a). Our western blot results confirmed that chaetocin inactivates the PI3K/ AKT pathway in GC cells (Fig. 5b). The chaetocininduced apoptotic cell death could be partially rescued by ectopic expression of constitutively active AKT (Fig. 5d, e). Consistently, the PI3K/AKT-specific inhibitor LY294002 enhanced chaetocin-induced cell apoptosis (Fig. 5f, g). Moreover, the expression of some antiapoptotic proteins (BCL-XL and MCL-1 of the BCL-2 family and XIAP of the IAP family) are regulated by the PI3K/AKT pathway ${ }^{37}$. Our results showed that both mRNA and protein levels of BCL-XL, MCL-1, and XIAP 


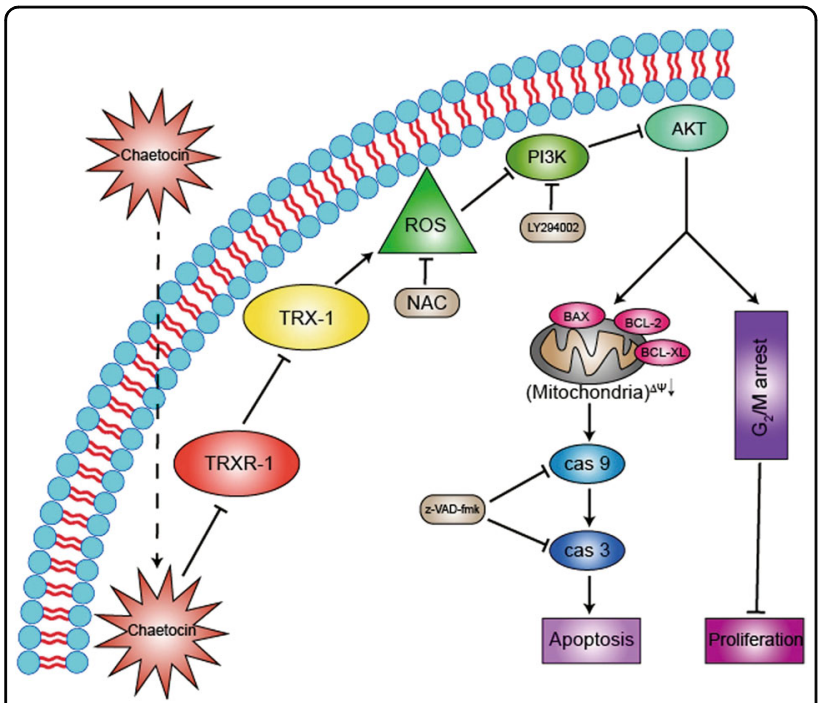

Fig. 8 The schematic diagram of possible molecular mechanism of chaetocin-induced cytotoxicity in GC

were downregulated in GC cells upon chaetocin treatment (Fig. 2c and Supplementary Fig. 4), indicating that the inhibition of the PI3K/AKT pathway by chaetocin may result in the downregulation of BCL-XL, MCL-1, and XIAP. These results suggest that the PI3K/AKT pathway is involved in chaetocin-induced GC cell apoptosis. As the ectopic expression of AKT could only partially rescue cells from apoptosis, there likely exist other pathways that are involved in chaetocin-induced cell death. Interestingly, when GC cells were cotreated with the ROS scavenger NAC, chaetocin-induced downregulation of $\mathrm{p}$ AKT was restored (Fig. 5h), suggesting that the chaetocininduced inactivation of AKT is regulated by ROS. Previous studies have shown that ROS can directly oxidize and dephosphorylate AKT, resulting in its inactivation ${ }^{38}$. However, no significant amount of oxidized AKT increased in GC cells treated with chaetocin, indicating chaetocin-induced ROS may not oxidize and inactivate AKT directly (Supplementary Fig. 6). Therefore, a more detailed investigation should be performed to determine the underlying mechanism of ROS-mediated regulation of the PI3K/AKT pathway in chaetocin-treated GC cells.

In summary, our data demonstrate that chaetocin inhibits TRXR-1 and subsequently induces excessive ROS accumulation followed by inactivation of the PI3K/AKT pathway, ultimately leading to GC cell death (Fig. 8). This suggests that chaetocin may be a potential candidate for anti-GC treatment.

\section{Acknowledgements}

This work was supported by the National Natural Science Foundation of China (81702418, 81672413); Guangdong Provincial Department of Science and Technology (2014B020212016, 2017A050501055, 2017A010105004);

Guangzhou Science Technology and Innovation Commission
(2016201604030007); Guangzhou Health and Medical Collaborative Innovation (15570006); China Postdoctoral Science Foundation (2017M612823); Overseas Excellent Professor Project, Ministry of Education, China; and National Key Clinical Discipline.

\section{Author details}

'Guangdong Provincial Key Laboratory of Colorectal and Pelvic Floor Diseases, Guangdong Institute of Gastroenterology, The Sixth Affiliated Hospital, Sun Yat-sen University, Guangzhou, Guangdong, China. ${ }^{2}$ Department of Gastrointestinal Surgery, The Sixth Affiliated Hospital, Sun Yat-sen University, Guangzhou, Guangdong, China. ${ }^{3}$ Department of Clinical Laboratory, The Sixth Affiliated Hospital, Sun Yat-sen University, Guangzhou, Guangdong, China. ${ }^{4}$ Guangdong Laboratory Animals Monitoring Institute, Guangdong Key Laboratory Animal Lab, Guangzhou, Guangdong, China. ${ }^{5}$ Dongguan Hospital of Southern Medical University, Guangzhou, Guangdong, China. ${ }^{6}$ School of Nursing, Sun Yat-sen University, Guangzhou, Guangdong, China

\section{Conflict of interest}

The authors declare that they have no conflict of interest.

\section{Publisher's note}

Springer Nature remains neutral with regard to jurisdictional claims in published maps and institutional affiliations.

Supplementary Information accompanies this paper at https://doi.org/ 10.1038/s41419-019-2035-x.

Received: 3 March 2019 Revised: 17 July 2019 Accepted: 2 August 2019 Published online: 24 October 2019

\section{References}

1. Bray, F. et al. Global Cancer Statistics 2018: GLOBOCAN estimates of incidence and mortality worldwide for 36 cancers in 185 countries. CA Cancer J. Clin. 68, 394-424 (2018).

2. Shah, M. A. Gastrointestinal cancer: targeted therapies in gastric cancer-the dawn of a new era. Nat. Rev. Clin. Oncol. 11, 10-11 (2014).

3. Van Cutsem, E., Sagaert, X., Topal, B., Haustermans, K. \& Prenen, H. Gastric cancer. Lancet 388, 2654-2664 (2016).

4. Bindoli, A. \& Rigobello, M. P. Principles in redox signaling: from chemistry to functional significance. Antioxid. Redox Signal. 18, 1557-1593 (2013).

5. Zhang, B. et al. Thioredoxin reductase inhibitors: a patent review. Expert Opin. Ther. Pat. 27, 547-556 (2017).

6. Rhee, S. G. \& Kil, I. S. Multiple functions and regulation of mammalian peroxiredoxins. Annu. Rev. Biochem. 86, 749-775 (2017).

7. Lillig, C. H. \& Holmgren, A. Thioredoxin and related molecules-from biology to health and disease. Antioxid. Redox Signal. 9, 25-47 (2007).

8. Kim, H. J. et al. Preferential elevation of prx I and trx expression in lung cancer cells following hypoxia and in human lung cancer tissues. Cell Biol. Toxicol. 19, 285-298 (2003).

9. Noike, T., Miwa, S., Soeda, J., Kobayashi, A. \& Miyagawa, S. Increased expression of thioredoxin-1, vascular endothelial growth factor, and redox factor-1 is associated with poor prognosis in patients with liver metastasis from colorectal cancer. Hum. Pathol. 39, 201-208 (2008).

10. Raffel, J. et al. Increased expression of thioredoxin-1 in human colorectal cancer is associated with decreased patient survival. J. Lab. Clin. Med. 142, 46-51 (2003)

11. Lim, J. Y. et al. Thioredoxin and thioredoxin-interacting protein as prognostic markers for gastric cancer recurrence. World J. Gastroenterol. 18, 5581-5588 (2012).

12. Hellfritsch, J. et al. Knockout of mitochondrial thioredoxin reductase stabilizes prolyl hydroxylase 2 and inhibits tumor growth and tumor-derived angiogenesis. Antioxid. Redox Signal. 22, 938-950 (2015).

13. Zou, P. et al. Piperlongumine as a direct TrxR1 inhibitor with suppressive activity against gastric cancer. Cancer Lett. 375, 114-126 (2016).

14. Smart, D. K. et al. Thioredoxin reductase as a potential molecular target for anticancer agents that induce oxidative stress. Cancer Res. 64, 6716-6724 (2004). 
15. Sekita, S. et al. Mycotoxin production by Chaetomium spp. and related fungi. Can. J. Microbiol. 27, 766-772 (1981).

16. Hauser, D., Weber, H. P. \& Sigg, H. P. Isolation and configuration of Chaetocin Helv. Chim. Acta 53, 1061-1073 (1970).

17. Liu, X., Guo, S., Liu, X. \& Su, L. Chaetocin induces endoplasmic reticulum stress response and leads to death receptor 5-dependent apoptosis in human nonsmall cell lung cancer cells. Apoptosis 20, 1499-1507 (2015).

18. Isham, C. R. et al. Chaetocin: a promising new antimyeloma agent with in vitro and in vivo activity mediated via imposition of oxidative stress. Blood 109, 2579-2588 (2007).

19. Isham, C. R., Tibodeau, J. D., Bossou, A. R., Merchan, J. R. \& Bible, K. C. The anticancer effects of chaetocin are independent of programmed cell death and hypoxia, and are associated with inhibition of endothelial cell proliferation. Brit. J. Cancer 106, 314-323 (2012).

20. Bae, J. S., Han, M., Yao, C. \& Chung, J. H. Chaetocin inhibits IBMX-induced melanogenesis in B16F10 mouse melanoma cells through activation of ERK. Chem. Biol. Interact. 245, 66-71 (2016).

21. Truitt, L., Hutchinson, C., DeCoteau, J. F. \& Geyer, C. R. Chaetocin antileukemia activity against chronic myelogenous leukemia cells is potentiated by bone marrow stromal factors and overcomes innate imatinib resistance. Oncogenesis 3, e122 (2014).

22. Lai, Y. S., Chen, J. Y., Tsai, H. J., Chen, T. Y. \& Hung, W. C. The SUV39H1 inhibitor chaetocin induces differentiation and shows synergistic cytotoxicity with other epigenetic drugs in acute myeloid leukemia cells. Blood Cancer J. 5, e313 (2015).

23. Lee, Y., Lim, J., Yoon, H., Chun, Y. \& Park, J. Antihepatoma activity of chaetocin due to deregulated splicing of hypoxia-inducible factor 1 alpha pre-mRNA in mice and in vitro. Hepatology 53, 171-180 (2011).

24. Greiner, D., Bonaldi, T., Eskeland, R., Roemer, E. \& Imhof, A. Identification of a specific inhibitor of the histone methyltransferase SU(VAR)3-9. Nat. Chem. Biol. 1, 143-145 (2005)

25. Tibodeau, J. D., Benson, L. M., Isham, C. R., Owen, W. G. \& Bible, K. C. The anticancer agent chaetocin is a competitive substrate and inhibitor of thioredoxin reductase. Antioxid. Redox Signal. 11, 1097-1106 (2009).

26. Murata, $\mathrm{H}$. et al. Glutaredoxin exerts an antiapoptotic effect by regulating the redox state of akt. J. Biol. Chem. 278, 50226-50233 (2003).

27. LO, Y. C., Ko, T. P., Su, W. C., Su, T. L. \& Wang, A. H. Terpyridine-platinum(II) complexes are effective inhibitors of mammalian topoisomerases and human thioredoxin reductase 1. J. Inorg. Biochem. 103, 1082-1092 (2009).

28. Ji, X. et al. Establishment and evaluation of four different types of patientderived xenograft models. Cancer Cell Int. 17, 122 (2017).

29. Crosio, C. et al. Mitotic phosphorylation of histoneh3: spatio-temporal regulation by mammalian aurora kinases. Mol. Cell. Biol. 22, 874-885 (2002).

30. Castedo, M. et al. Cell death by mitotic catastrophe: a molecular definition. Oncogene 23, 2825-2837 (2004).

31. Gandin, V. et al. Cancer cell death induced by phosphine gold $(I)$ compounds targeting thioredoxin reductase. Biochem. Pharmacol. 79, 90-101 (2010).

32. Zou, P. et al. Auranofin induces apoptosis by ROS-mediated ER stress and mitochondrial dysfunction and displayed synergistic lethality with piperlongumine in gastric cancer. Oncotarget 6, 36505-36521 (2015).

33. Chaib, $H$. et al. Anti-leukemia activity of chaetocin via death receptordependent apoptosis and dual modulation of the histone methyl-transferase SUV39H1. Leukemia 26, 662-674 (2012).

34. Dixit, D., Ghildiyal, R., Anto, N. P. \& Sen, E. Chaetocin-induced ROS-mediated apoptosis involves ATM-YAP1 axis and JNK-dependent inhibition of glucose metabolism. Cell Death Dis. 5, e1212 (2014).

35. Leslie, N. R. The redox regulation of PI 3-kinase-dependent signaling. Antioxid. Redox Signal. 8, 1765-1774 (2006).
36. Liu, P., Cheng, H., Roberts, T. M. \& Zhao, J. J. Targeting the phosphoinositide 3kinase pathway in cancer. Nat. Rev. Drug Discov. 8, 627-644 (2009).

37. Hers, I., Vincent, E. E. \& Tavare, J. M. Akt signalling in health and disease. Cell. Signal. 23, 1515-1527 (2011).

38. Cao, J. et al. ROS-driven Akt dephosphorylation at Ser-473 is involved in 4-HPRmediated apoptosis in NB4 cells. Free Radic. Biol. Med. 47, 536-547 (2009).

39. Holmstrom, K. M. \& Finkel, T. Cellular mechanisms and physiological consequences of redox-dependent signalling. Nat. Rev. Mol. Cell Biol. 15, 411-421 (2014).

40. D'Autreaux, B. \& Toledano, M. B. ROS as signalling molecules: mechanisms that generate specificity in ROS homeostasis. Nat. Rev. Mol. Cell Biol. 8, 813-824 (2007).

41. Roy, J., Galano, J. M., Durand, T., Le Guennec, J. Y. \& Lee, J. C. Physiological role of reactive oxygen species as promoters of natural defenses. Faseb J. 31, 3729-3745 (2017)

42. Reuter, S., Gupta, S. C., Chaturvedi, M. M. \& Aggarwal, B. B. Oxidative stress, inflammation, and cancer: how are they linked? Free Radic. Biol. Med. 49, 1603-1616 (2010).

43. Tafani, M. et al. The interplay of reactive oxygen species, hypoxia, inflammation, and sirtuins in cancer initiation and progression. Oxid. Med. Cell. Longev. 2016, 3907147 (2016).

44. Galadari, S., Rahman, A., Pallichankandy, S. \& Thayyullathil, F. Reactive oxygen species and cancer paradox: to promote or to suppress? Free Radic. Biol. Med. 104, 144-164 (2017).

45. Weng, M. S., Chang, J. H., Hung, W. Y., Yang, Y. C. \& Chien, M. H. The interplay of reactive oxygen species and the epidermal growth factor receptor in tumor progression and drug resistance. J. Exp. Clin. Cancer Res. 37, 61 (2018).

46. Li, $Y$. et al. Cardamonin induces ROS-mediated G2/M phase arrest and apoptosis through inhibition of NF-kB pathway in nasopharyngeal carcinoma. Cell Death Dis. 8, e3024 (2017).

47. Jiang, Y., Wang, X. \& Hu, D. Furanodienone induces G0/G1 arrest and causes apoptosis via the ROS/MAPKs-mediated caspase-dependent pathway in human colorectal cancer cells: a study in vitro and in vivo. Cell Death Dis. 8, e2815 (2017).

48. Kondo, N., Nakamura, H., Masutani, H. \& Yodoi, J. Redox regulation of human thioredoxin network. Antioxid. Redox Signal. 8, 1881-1890 (2006).

49. Grogan, T. M. et al. Thioredoxin, a putative oncogene product, is overexpressed in gastric carcinoma and associated with increased proliferation and increased cell survival. Hum. Pathol. 31, 475-481 (2000).

50. Millet, R. et al. Synthesis of 5-nitro-2-furancarbohydrazides and their cisdiamminedichloroplatinum complexes as bitopic and irreversible human thioredoxin reductase inhibitors. J. Med. Chem. 48, 7024-7039 (2005).

51. Sasada, T. et al. Possible involvement of thioredoxin reductase as well as thioredoxin in cellular sensitivity to cis-diamminedichloroplatinum (II). Free Radic. Biol. Med. 27, 504-514 (1999).

52. Zhong, L. \& Holmgren, A. Essential role of selenium in the catalytic activities of mammalian thioredoxin reductase revealed by characterization of recombinant enzymes with selenocysteine mutations. J. Biol. Chem. 275, 18121-18128 (2000).

53. Cai, W. et al. Small molecule inhibitors of mammalian thioredoxin reductase. Free Radic. Biol. Med. 52, 257-265 (2012).

54. Cherblanc, F. L., Chapman, K. L., Brown, R. \& Fuchter, M. J. Chaetocin is a nonspecific inhibitor of histone lysine methyltransferases. Nat. Chem. Biol. 9, 136-137 (2013).

55. Li, X. et al. Synergistic antitumor activity of withaferin A combined with oxaliplatin triggers reactive oxygen species-mediated inactivation of the PI3KAKT pathway in human pancreatic cancer cells. Cancer Lett. 357, 219-230 (2015). 